\title{
A Throughput Model of IEEE 802.11aa Intra-Access Category Prioritization
}

\author{
Katarzyna Kosek-Szott
}

Published online: 29 September 2012

(C) The Author(s) 2012. This article is published with open access at Springerlink.com

\begin{abstract}
In this paper the first saturation throughput model of an IEEE 802.11 network is presented, which includes the intra-access category prioritization introduced in the recently approved IEEE 802.11aa standard. This new feature was proposed to support finer grained prioritization of audio video streams in comparison to the existing Enhanced Distributed Channel Access (EDCA) function. The presented model implements different transmission probabilities for the primary and alternate voice and video queues. Additionally, it includes such features as virtual collision handling, backoff differentiation, and Arbitration InterFrame Space differentiation. The presented results show the difference in the operation of IEEE 802.11aa intra-access category prioritization and EDCA inter-access category prioritization. They also allow to derive several novel conclusions.
\end{abstract}

Keywords Intra-access category prioritization · QoS · IEEE 802.11 aa ·

Audio video streaming

\section{Introduction}

The new 802.11aa standard has recently been approved by IEEE [2]. It defines a number of enhancements to IEEE 802.11 to allow robust audio and video streaming for consumer and enterprise applications. These enhancements include: groupcast with retries (GCR), stream classification service (SCS), overlapping basic service set (OBSS) management, interworking with the IEEE 802.1Q stream reservation protocol (SRP), and intra-access category (intra-AC) prioritization. This last feature is investigated in this paper.

Intra-AC prioritization is an extension of the Enhanced Distributed Channel Access (EDCA) function defined in the IEEE 802.11 standard [1]. Instead of only four transmit

\footnotetext{
K. Kosek-Szott $(\bowtie)$

Faculty of Computer Science, Electronics and Telecomunications, AGH University of Science and Technology, Al. Mickiewicza 30, 30-059 Krakow, Poland e-mail: kosek@kt.agh.edu.pl
} 


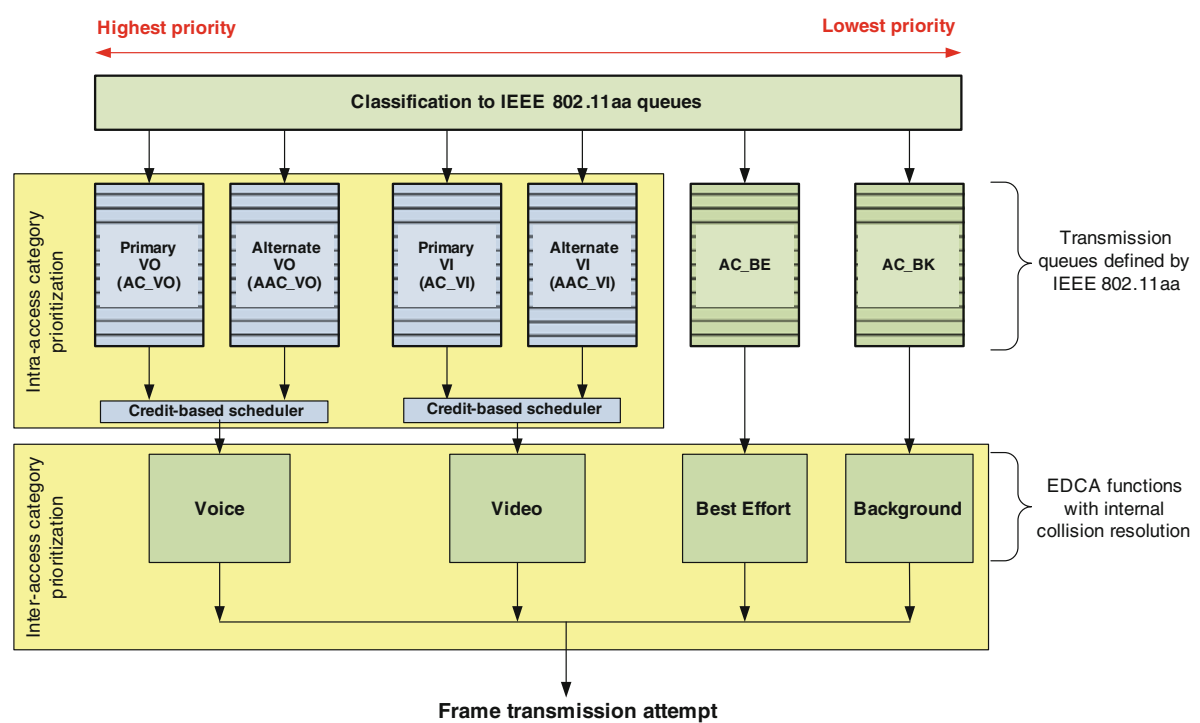

Fig. 1 Intra-AC traffic prioritization defined by the IEEE 802.11aa standard

queues, six of them are used to provide finer grained prioritization between individual audio and video streams (Fig. 1). They are defined as follows: two queues for voice traffic (primary-AC_VO and alternate-AAC_VO), two queues for video traffic (primaryAC_VI and alternate-AAC_VI), one queue for best effort traffic (AC_BE), and one queue for background traffic (AC_BK). These transmit queues are mapped to four independent EDCA functions (EDCAF) to enable traffic differentiation over the wireless channel (Fig. 1) using four EDCA Access Categories (ACs): VO, VI, BE, and BK [1]. The head-of-line frames belonging to $\mathrm{AC}_{-} \mathrm{VO}$ and $\mathrm{AAC}$-VO (AC_VI and $\mathrm{AAC}_{-} \mathrm{VI}$ ) are passed to $\mathrm{VO}$ EDCAF (VI EDCAF) according to a credit-based scheduler (with two queues) as defined in IEEE 802.1Qav [3]. Additionally, the IEEE 802.11aa standard specifies that, for voice and video streams, frames belonging to the primary queues are selected with a higher probability than frames from the alternate queues.

In the literature, to the author's best knowledge, no analytical model including the intraAC prioritization feature of IEEE 802.11 aa has thus far been proposed. Therefore, this paper presents the first such model. It is based on a previous comprehensive EDCA model [4]. The modelling of BE and BK traffic remains the same as proposed in [4] while for VO and VI traffic two additional transmit queues are considered.

\section{Analytical Model}

For each AC, let $s(t), b(t)$, and $c(t)$ denote the stochastic processes representing the backoff stage, the backoff counter, and the remaining number of time slots during the deferring period at time $t$, respectively. The 3-D process $\{s(t), \mathrm{b}(t), \mathrm{c}(t)\}$ can be modelled as discrete-time Markov chains shown in Fig. 2 (for BE and BK) and in Fig. 3 (for VO and VI). The parameter $d$ in Fig. 2 indicates the differing period, i.e., the difference in the number of time slots between the minimum AIFS (AIFS min $_{\text {) }}$ and the AIFS of the $v$ th AC (for EDCA $v \in\{0,1,2$, 3 \}, which corresponds to BK, BE, VI, and VO, respectively). In Fig. 3, since both VO and 


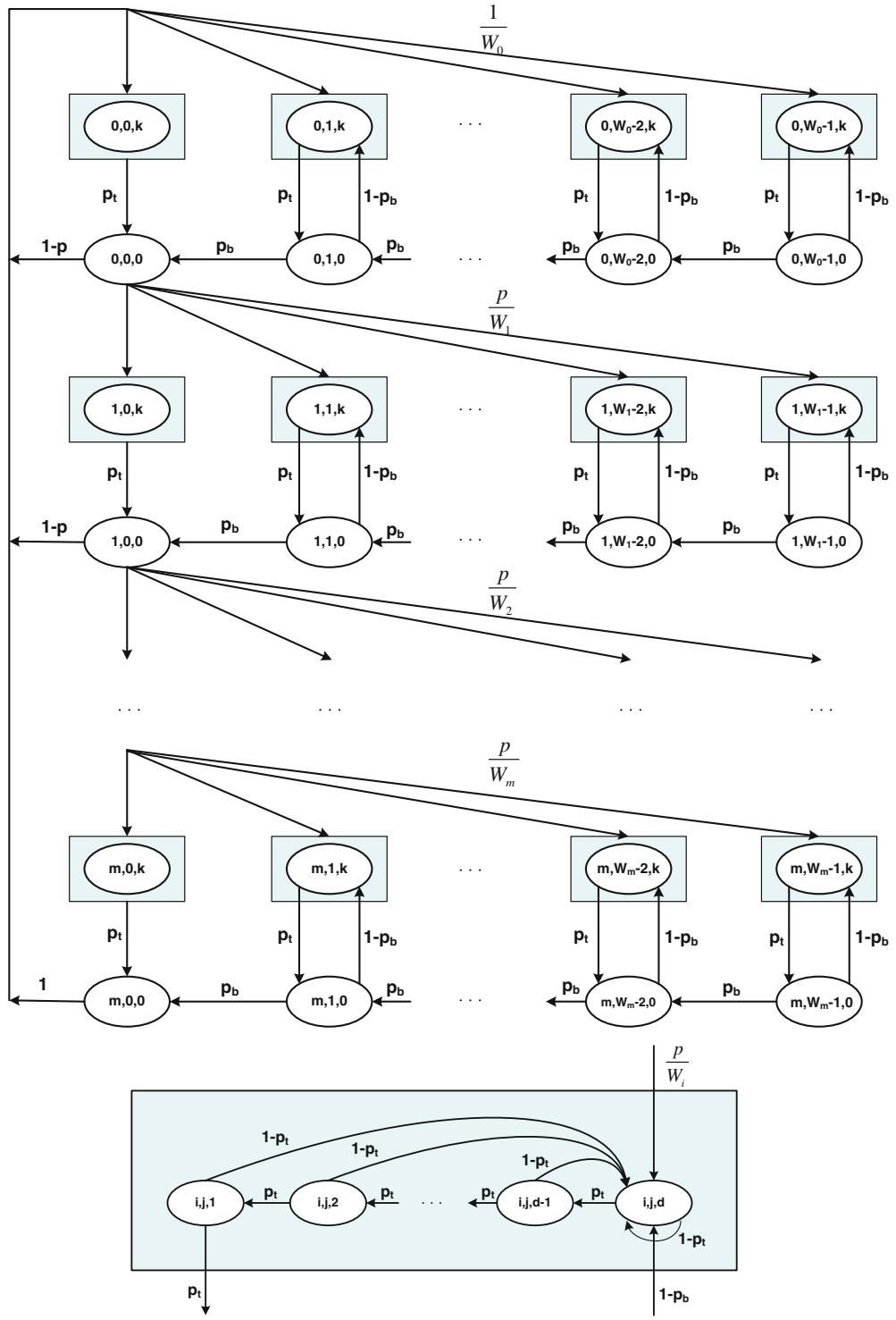

Fig. 2 Markov chain for BE $(v=1)$ and BK $(v=0)$ ACs

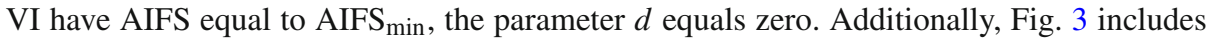
the intra-AC feature of the IEEE 802.11aa standard in the form of different transmission probabilities for the primary and alternate queues ( $p_{v}^{p}$ and $p_{v}^{a}$, respectively).

Let $b_{i, j, k}$ be the stationary distribution of the proposed Markov chains. Then, based on the chain regularities the steady state probabilities for the $v$ th $\mathrm{AC}$ can be calculated:

$$
\begin{aligned}
b_{i, 0,0} & =p_{v}^{i} b_{0,0,0}, \quad 0 \leq i \leq m, \\
b_{i, j, 0} & =\frac{W_{i v}-j}{p_{b v} W_{i v}} p_{v}^{i} b_{0,0,0}, \quad 0 \leq i \leq m, 1 \leq j \leq W_{i v}-1,
\end{aligned}
$$




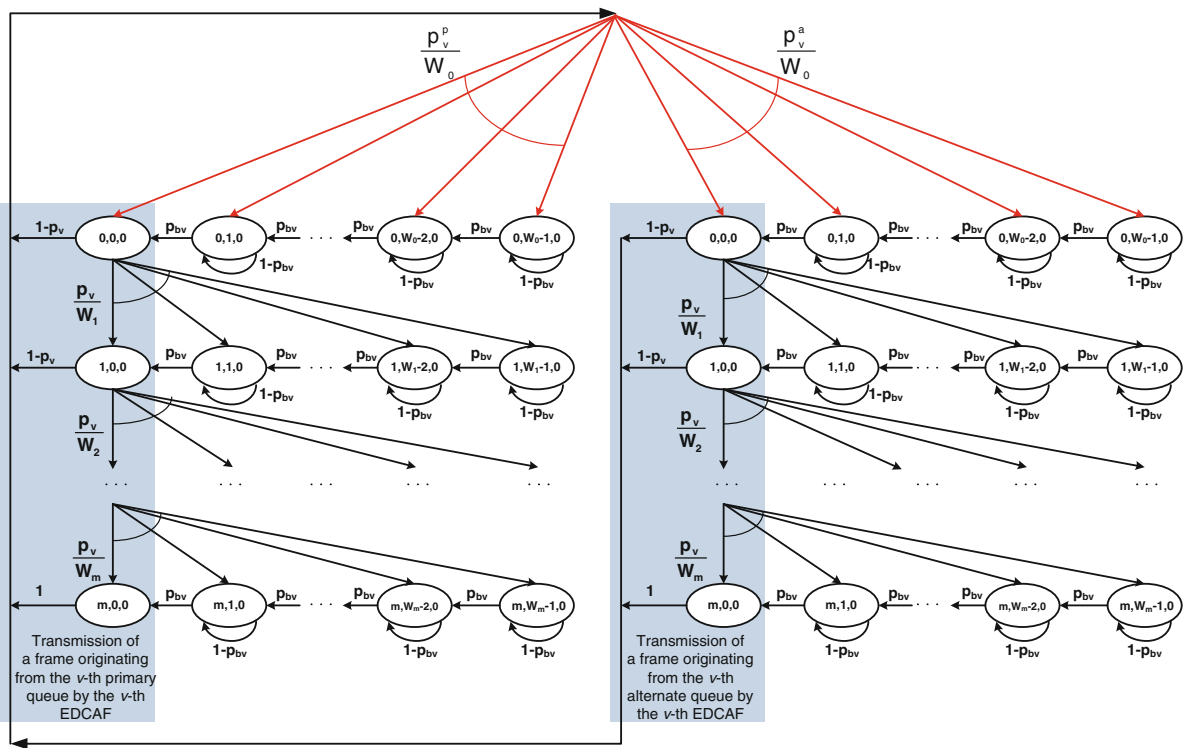

Fig. 3 Markov chain for VO $(v=3)$ and VI $(v=2)$ ACs including the intra-AC prioritization feature of the IEEE 802.11aa standard

where $m$ is the frame retransmission limit, $W_{i v}-1$ is the size of the contention window after the $i$ th retransmission, $p_{v}$ is the probability of collision, and $p_{b v}$ is the probability of an idle medium in a time slot after AIFS.

From Fig. 2 and from the normalization condition, for BE and BK we have:

$$
\begin{aligned}
& b_{i, j, k}=\frac{b_{i, 0,0}}{p_{t v}^{k} W_{i v}}+\frac{1-p_{b v}}{p_{t v}^{k}} b_{i, j, 0}, \quad 0 \leq i \leq m, 1 \leq j \leq W_{i v}-1,1 \leq k \leq d, \\
& \sum_{i=0}^{m} b_{i, 0,0}+\sum_{i=0}^{m} \sum_{j=1}^{W_{i v}-1} b_{i, j, 0}+\sum_{i=0}^{m} \sum_{j=1}^{W_{i v}-1} \sum_{k=1}^{d_{v}} b_{i, j, k}=1 .
\end{aligned}
$$

From the normalization condition for VO and VI we get (Fig. 3):

$$
\sum_{i=0}^{m} b_{i, 0,0}+\sum_{i=0}^{m} \sum_{j=1}^{W_{i v}-1} b_{i, j, 0}=1
$$

Equations (1)-(4) allow us to calculate $b_{0,0,0}$ for BE and BK:

$$
\begin{aligned}
b_{0,0,0}= & {\left[\frac{\left(1-p_{t v}^{d_{v}}\right)}{\left(1-p_{t v}\right) p_{t v}^{d_{v}}}\left(\left(1-p_{b v}\right) \sum_{i=0}^{m} \frac{W_{i v}-1}{2 p_{b v}} p_{v}^{i}+\sum_{i=0}^{m} \frac{p_{v}^{i}}{W_{i v}}\right)\right.} \\
& \left.+\sum_{i=0}^{m} \frac{W_{i v}-1}{2 p_{b v}} p_{v}^{i}+\frac{1-p_{v}^{m+1}}{1-p_{v}}\right]^{-1}
\end{aligned}
$$


Equations (1), (2), and (5) allow us to calculate $b_{0,0,0}$ for VO and VI:

$$
b_{0,0,0}=\left[\sum_{i=0}^{m} \frac{W_{i v}-1}{2 p_{b v}} p_{v}^{i}+\frac{1-p_{v}^{m+1}}{1-p_{v}}\right]^{-1}
$$

For a network with $n$ stations, the following probabilities are additionally defined:

1. the overall transmission probability for the $v$ th $\mathrm{AC}: \tau_{v}=\frac{\left(1-p_{v}^{m+1}\right)}{1-p_{v}} b_{0,0,0}$,

2. the transmission probability of a frame from the primary queue of the VO and VI AC: $\tau_{v}^{p}=p_{v}^{p} \tau_{v}$, where $p_{v}^{p}$ is the probability of selecting the head-of-line frame from the primary queue of the $v$ th AC to be passed to the $v$ th EDCAF, ${ }^{1}$

3. the transmission probability of a frame from the alternate queue of the VO and VI AC: $\tau_{v}^{a}=p_{v}^{a} \tau_{v}$, where $p_{v}^{a}$ is the probability of selecting the head-of-line frame from the alternate queue of the $v$ th AC to be passed to the $v$ th EDCAF, additionally it is assumed that under saturation $p_{v}^{a}$ equals $1-p_{v}^{p}$,

4. the probability that after AIFS the wireless channel is idle: $p_{b v}=\prod_{x=0, x \neq v}^{3}\left(1-\tau_{x}\right)^{n}$ $\left(1-\tau_{v}\right)^{n-1}$,

5. the probability of virtual and/or physical collisions: $p_{v}=1-\prod_{x \leq v}\left(1-\tau_{x}\right)^{n-1}$ $\prod_{x>v}\left(1-\tau_{x}\right)^{n}, 0 \leq x \leq 3$,

6. probability of an idle channel in a time slot during the deferring period $d p_{t v}=$ $\prod_{x>v}\left(1-\tau_{x}\right)^{n}, 0 \leq x \leq 3$.

For each $\mathrm{AC}$, we define the throughput as:

$$
T H_{v}=\frac{P S_{v} L}{(1-P T) \delta+\sum_{x=0}^{N-1} P S_{x} T_{S x}+\left(P T-\sum_{x=0}^{N-1} P S_{x}\right) T_{c v}},
$$

where $L$ is the expected time spent on transmitting a DATA frame, $P S_{v}$ is the probability of a successful transmission for the $v$ th AC: $P S_{v}=n \tau_{v} \prod_{x \leq v}\left(1-\tau_{v}\right)^{n-1} \prod_{x>v}\left(1-\tau_{v}\right)^{n}$; $P T$ is the probability that at least one AC transmits in a time slot: $P T=1-\prod_{v=0}^{3}\left(1-\tau_{v}\right)^{n} ; \delta$ is the time duration of one time slot, $T_{c x}\left(T_{s v}\right)$ is the average time of a collision (successful transmission) for the $x$ th ( $v$ th) AC.

$T_{s v}$ and $T_{c v}$ depend on the channel access method used. For the basic channel access we have:

$$
\begin{gathered}
T_{s v}=\mathrm{H}+L+\mathrm{SIFS}+\mathrm{ACK}+\text { AIFS }_{\min }+2 \times \varepsilon, \\
T_{c v}=\mathrm{H}+L+\varepsilon+\mathrm{EIFS},
\end{gathered}
$$

where $H$ is the time occupied by the PHY and MAC headers, $\varepsilon$ is the propagation delay, SIFS is the Short Inter-Frame Space, and ACK is the time occupied by an ACK transmission. For the four-way handshake mechanism we have:

$$
\begin{gathered}
T_{s v}=\mathrm{RTS}+\mathrm{CTS}+\mathrm{H}+L+\mathrm{ACK}+\mathrm{AIFS}_{\min }+3 \times \mathrm{SIFS}+4 \times \varepsilon, \\
T_{c v}=\mathrm{RTS}+\mathrm{EIFS},
\end{gathered}
$$

1 This probability is directly based on the parameter configuration of the credit-based scheduler. However, the exact configuration has not been specified in the IEEE 802.11 aa standard. 
where RTS and CTS is the time required to send the Request To Send (RTS) and Clear To Send (CTS) frames, respectively.

Additionally, for VO and VI, we can calculate the average saturation throughput for frames belonging to the primary queues defined by IEEE 802.11aa:

$$
T H_{v}^{p}=\frac{P S_{v}^{p} L}{(1-P T) \delta+\sum_{x=0}^{3} P S_{x} T_{s x}+\left(P T-\sum_{x=0}^{3} P S_{x}\right) T_{c v}},
$$

where $P S_{v}^{p}$ is the probability of a successful transmission for the $v$ th AC of frames belonging to the primary queue:

$$
P S_{v}^{p}=n \tau_{v}^{p} \prod_{x \leq v}\left(1-\tau_{v}\right)^{n-1} \prod_{x>v}\left(1-\tau_{v}\right)^{n}, \quad 0 \leq x \leq 3
$$

Consequently, the average saturation throughput for frames belonging to the alternate VO and VI queues defined by IEEE 802.11aa is:

$$
T H_{v}^{a}=\frac{P S_{v}^{a} L}{(1-P T) \delta+\sum_{x=0}^{3} P S_{x} T_{s x}+\left(P T-\sum_{x=0}^{3} P S_{x}\right) T_{c v}},
$$

where $P S_{v}^{a}$ is the probability of a successful transmission for the $v$ th AC of frames belonging to the alternate queue:

$$
P S_{v}^{a}=n \tau_{v}^{a} \prod_{x \leq v}\left(1-\tau_{v}\right)^{n-1} \prod_{x>v}\left(1-\tau_{v}\right)^{n}, \quad 0 \leq x \leq 3 .
$$

Finally, the overall saturation throughput for the VO and VI ACs is:

$$
T H_{v}=T H_{v}^{p}+T H_{v}^{a},
$$

and it is equal to the throughput defined in (8).

\section{Theoretical and Simulation Results}

The proposed IEEE 802.11aa model was compared with simulations. The calculations were done in Wolfram Mathematica 8.0 [5] and the simulations were performed using an extended version of the ns-2 simulator [6]. IEEE 802.11b was chosen as the PHY layer and the standard EDCA parameters were set at the MAC layer [1]. The DATA frame size was set to 1000 bytes. Nodes were placed randomly, saturation conditions were assumed for each AC, and hidden nodes were avoided. Finally, the simulations were repeated until the error of each simulation point was smaller than $3 \%$ assuming $95 \%$ confidence intervals.

Table 1 presents a comparison of simulated and theoretical throughput values for voice and video EDCA ACs, as well as their corresponding primary and alternate IEEE 802.11aa queues. Two networks were analyzed: one composed of 10 nodes and the second composed of 20 nodes. In both cases each node transmitted traffic belonging to all four ACs. Additionally, different values of $p_{v}^{p}$ and $p_{v}^{a}$ were chosen for different priority queues in each of the analyzed networks. A close matching between the theoretical and simulated throughput values was achieved.

Figure 4 constitutes a graphical representation of the gathered theoretical results for both analyzed networks. The following conclusions can be derived. Firstly, prioritization between 
Table 1 Comparison of simulated and theoretical normalized throughput values for configuration 1 (10 node network) and configuration 2 (20 node network)

Normalized throughput (\%)

\begin{tabular}{llllllll}
\hline EDCA & EDCA & EDCA & EDCA & $802.11 \mathrm{aa}$ & $802.11 \mathrm{aa}$ & $802.11 \mathrm{aa}$ & $802.11 \mathrm{aa}$ \\
AC VO & AC VI & AC BE & AC BK & $\begin{array}{l}\text { AC_VO } \\
\left(p_{3}^{p}=0.75\right)\end{array}$ & $\begin{array}{l}\text { AAC_VO } \\
\left(p_{3}^{a}=0.25\right)\end{array}$ & $\begin{array}{l}\text { AC_VI } \\
\left(p_{2}^{p}=0.80\right)\end{array}$ & $\begin{array}{l}\text { AAC_VI } \\
\left(p_{2}^{a}=0.20\right)\end{array}$ \\
\hline
\end{tabular}

Configuration 1

\begin{tabular}{|c|c|c|c|c|c|c|c|c|}
\hline Model & 23.14 & 10.24 & 0.82 & 0.01 & 17.36 & 5.79 & 8.19 & 2.05 \\
\hline Simulation & 26.17 & 7.99 & 0.14 & 0.01 & 19.63 & 6.54 & 6.39 & 1.60 \\
\hline & EDCA & EDCA & EDCA & EDCA & $802.11 \mathrm{aa}$ & $802.11 \mathrm{aa}$ & $802.11 \mathrm{aa}$ & $802.11 \mathrm{aa}$ \\
\hline & $\mathrm{AC} \mathrm{VO}$ & AC VI & $\mathrm{AC} B E$ & AC BK & $\begin{array}{l}\mathrm{AC}_{-} \mathrm{VO} \\
\left(p_{3}^{p}=0.70\right)\end{array}$ & $\begin{array}{l}\text { AAC_VO } \\
\left(p_{3}^{a}=0.30\right)\end{array}$ & $\begin{array}{l}\mathrm{AC}_{-} \mathrm{VI} \\
\left(p_{2}^{p}=0.60\right)\end{array}$ & $\begin{array}{l}\text { AAC_VI } \\
\left(p_{2}^{a}=0.40\right)\end{array}$ \\
\hline
\end{tabular}

\section{Configuration 2}

\begin{tabular}{lllllllll} 
Model & 18.82 & 8.60 & 0.43 & 0.00 & 13.17 & 5.65 & 5.16 & 3.44 \\
Simulation & 20.06 & 6.93 & 0.03 & 0.00 & 14.04 & 6.02 & 4.16 & 2.77 \\
\hline
\end{tabular}

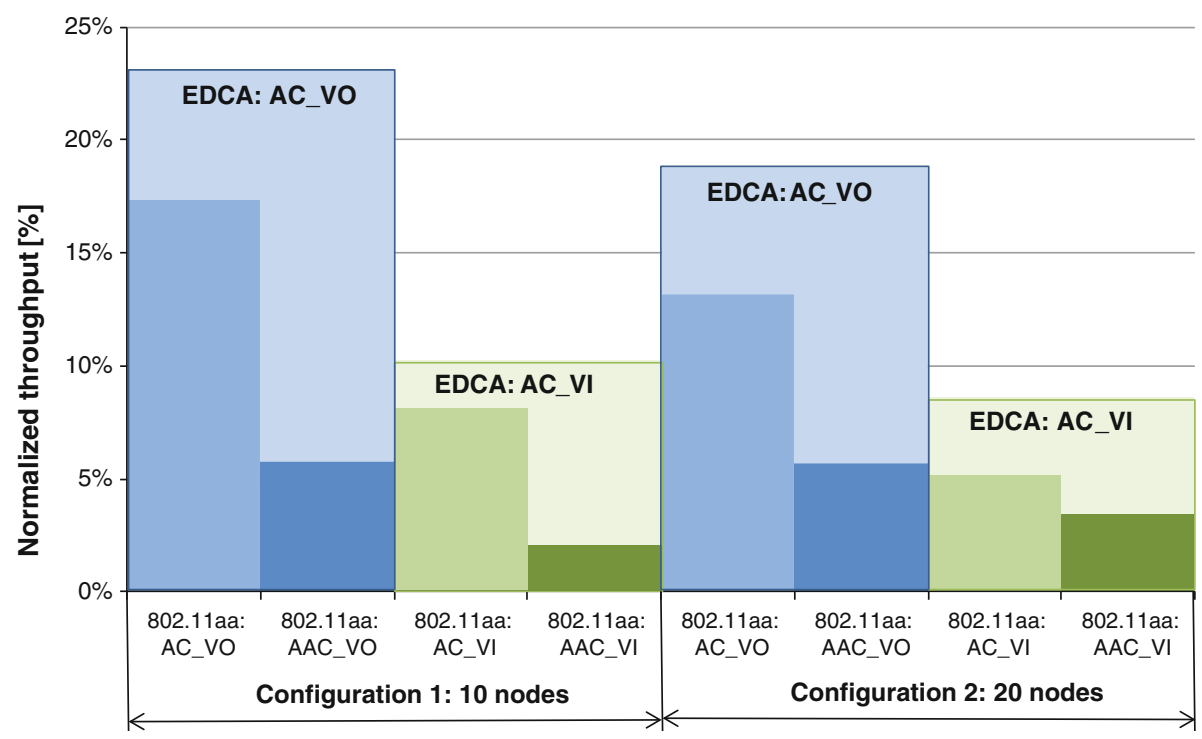

Fig. 4 Normalized throughput comparison for primary and alternate IEEE 802.11aa VO and VI queues as well as for EDCA VO and VI ACs

AC VO and AC VI (i.e., inter-AC prioritization) is achieved. Furthermore, if frames are chosen with higher probability from the primary queues $\left(p_{3}^{p}, p_{2}^{p}\right)$ than from alternate queues $\left(p_{3}^{a}, p_{2}^{a}\right)$, as defined by the IEEE 802.11 aa standard, then adequate intra-access category prioritization between primary and alternate queues is also achieved. Interestingly, for certain values of $p_{3}^{a}$ frames belonging to the primary VI queue can achieve higher throughput than those belonging to the alternate VO queue (c.f., configuration 1 in Fig. 4). In such a case the majority of frames are selected from the VO primary queue $(75 \%)$ and, therefore, the 
probability $p_{3}^{a}$ is small (equal to 0.25 ). At the same time, a larger number of frames is selected from the VI alternate queue $(80 \%)$ than from the VO alternate queue $(25 \%)$, i.e., $p_{2}^{a}>p_{3}^{a}$. Therefore, to assure strict prioritization of VO streams over VI streams under saturation such configurations should be avoided. Finally, different networks can be served with different prioritization patterns which was not possible when only EDCA inter-AC prioritization procedures were applied (c.f., configuration 1 vs. configuration 2 in Fig. 4).

\section{Conclusions}

In this paper the first analytical saturation throughput model of the intra-AC prioritization feature of the IEEE 802.11aa standard was described. The presented results show that the new IEEE 802.11aa intra-AC prioritization feature provides a finer grained prioritization of VO and VI streams, in comparison to the currently used EDCA inter-AC prioritization. Additionally, it was shown that the proposed model can be used to analyze and compare networks in which the intra-AC and inter-AC prioritization are applied.

Acknowledgments This work has been carried out as part of a project financed by the National Science Centre (decision no. DEC-2011/01/D/ST7/05166).

Open Access This article is distributed under the terms of the Creative Commons Attribution License which permits any use, distribution, and reproduction in any medium, provided the original author(s) and the source are credited.

\section{References}

1. IEEE Std 802.11-2012 (2012). IEEE Standard for Information technology-telecommunications and information exchange between systems - local and metropolitan area networks - specific requirementspart 11: Wireless LAN Medium Access Control (MAC) and Physical Layer (PHY) Specifications, March (2012).

2. IEEE 802.11aa (2012). IEEE standard for local and metropolitan area networks-specific requirementspart 11: Wireless LAN medium access control (MAC) and physical layer (PHY) specifications-amendment 2: MAC enhancements for Robust 12 Audio Video Streaming, May (2012).

3. IEEE Std 802.1Qav-2009 (2010). IEEE standard for local and metropolitan area networks-Virtual bridged local area networks. Amendment 12: Forwarding and queuing enhancements for time-sensitive streams, January (2010).

4. Gas, M., Kosek-Szott, K., Natkaniec, M., \& Pach, A. R. (2011). 3D Markov chain-based saturation throughput model of IEEE 802.11 EDCA. Electronics Letters, 47(14), 826-827.

5. Mathematica 8.0 [Online] http://www.wolfram.com.

6. The Network Simulator ns-2. [Online] http://isi.edu/nsnam/ns. 


\section{Author Biography}

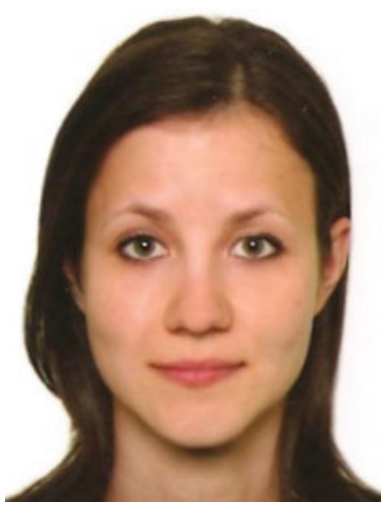

Katarzyna Kosek-Szott received her M.Sc. and Ph.D. degrees in telecommunications (both with honours) from the AGH University of Science and Technology, Krakow, Poland in 2006 and 2011, respectively. Currently she is working as an assistant professor at the Department of Telecommunications, AGH University of Science and Technology. Her general research interests are focused on wireless networking. The major topics include wireless LANs (especially ad-hoc networks) and quality of service provisioning within these networks. She is a reviewer for international journals and conferences. She has been involved in several European projects: DAIDALOS II, CONTENT, CARMEN, FLAVIA as well as grants supported by the Polish Ministry of Science and Higher Education. She has also co-authored a number of research papers. 